ИЗВЕСТИЯ АКАДЕМИИ НАУК ЭСТОНСКОИ ССР. ФИЗИКА * МАТЕМАТИКА

PROCEEDINGS OF THE ACADEMY OF SCIENCES OF THE ESTONIAN SSR. PHYSICS * MATHEMATICS

$1987,36,3$

УДК 519.226 .3

B. ОЛЬМАН

\title{
СОХРАНЕНИЕ БАИЕСОВСКОГО СВОЙСТВА ОТНОСИТЕЛЬНО ДИСКРЕТНЫХ МЕР ПРИ ПРЕОБРАЗОВАНИЯХ
}

\author{
(Представил Н. Алумяэ)
}

В теории статистического оценивания часто возникает необходимость оценивать не сам неизвестный параметр распределения $\theta$, а некоторую функцию $f(\theta)$ от него. Так, например, в теории надежности обычно требуется оценивать по наблюдениям не интенсивность отказов $\theta$, а вероятность безотказной работы в течение времени $T$, т. е. величину $e^{-\theta T}$. Естественно, возникает вопрос: для каких преобразований $f$ некоторое заданное статистическое свойство оценки $\hat{\theta}$ параметра $\theta$ сохраняется при оценивании $f(\theta)$ с помощью оценки $f(\theta)$ ? Известно, например, что если $\hat{\theta}$ - оценка максимального правдоподобия (ОМП), то для широкого класса функций $f(\hat{\theta})$ является ОМП величины $f(\theta)$. В настоящем сообщении исследуется сохранение байесовского свойства относительно дискретных мер и показано, что только линейные преобразования сохраняют байесовость.

Рассмотрим задачу оценивания функции $f(\theta,) \theta \in R^{p}, p \geqslant 1$, по наблюдению $x \in X$ при условии, что плотность $p(x, \theta)$ распределения $x$ относительно некоторой меры $d \mu(x)$ известна с точностью до параметра $\theta$ и что $f: R^{p \rightarrow} R^{r}, r \geqslant 1$. Неизвестный параметр $\theta$ является случайным независимым от $x$ вектором, подчиненным некоторому вероятностному распределению $G$. При квадратичной потере риск произвольной оценки $\tilde{\theta}(x)$ определяется интегралом

$$
\int_{\theta \in R^{p}} \int_{x \in X}(\tilde{\theta}(x)-\theta)^{2} p(x, \theta) d \mu(x) d G(\theta)
$$

и минимизирующая этот функционал байесовская оценка $\theta_{G}(x)$ имеет вид

$$
\theta_{G}(x)=\int_{\theta \in R^{p}} \theta p(x, \theta) d G(\theta) / \int_{\theta \in R^{p}} p(x, \theta) d G(\theta) .
$$

О п р ед елен и е. Назовем функцию $f$ сохраняющей байесовость оценки $\theta_{G}$, если статистика $f\left(\theta_{G}(x)\right)$ является байесовской оценкой $f(\theta)$ относительно распределения $G$.

В соответствии с определением $f$ сохраняет байесовость $\theta_{G}$, если для почти всех $x$ по мере $d \mu(x)$ имеет место равенство

$$
f\left(\theta_{G}(x)\right)=\int_{\theta \in R^{p}} f(\theta) p(x, \theta) d G(\theta) / \int_{\theta \in R^{p}} p(x, \theta) d G(\theta) .
$$

Пусть распределение $G$ сосредоточено на конечном множестве векторов $\Theta=\left\{\theta_{1}, \theta_{2}, \ldots, \theta_{n}\right\}, \theta_{i} \in R^{p}$, с нагрузками $p_{1}, p_{2}, \ldots, p_{n}, n \geqslant$. Тогда равенство (1) имеет вид 


$$
f\left(\frac{\sum_{i=1}^{n} \theta_{i} p\left(x, \theta_{i}\right) p_{i}}{\sum_{i=1}^{n} p\left(x, \theta_{i}\right) p_{i}}\right)=\frac{\sum_{i=1}^{n} f\left(\theta_{i}\right) p\left(x, \theta_{i}\right) p_{i}}{\sum_{i=1}^{n} p\left(x, \theta_{i}\right) p_{i}}
$$

Или, обозначив $q_{i}(x)=p\left(x, \theta_{i}\right) p_{i} / \sum_{i=1}^{n} p_{i} p\left(x, \theta_{i}\right)$, имеем

$$
f\left(\sum_{i=1}^{n} \theta_{i} q_{i}(x)\right)=\sum_{i=1}^{n} f\left(\theta_{i}\right) q_{i}(x)
$$

Пусть $\theta_{G}(X)=\Theta_{0}-$ множество значений статистики $\theta_{G}$. Произвол в значениях $f\left(\theta_{i}\right), i=1,2, \ldots, n$, удовлетворяющих уравнению (2), определяется пересечением $\Theta_{0} \cap \Theta$. В частности, если $\Theta_{0} \cap \Theta=\varnothing$, то $f\left(\theta_{i}\right)$, $i=1,2, \ldots, n$, могут быть произвольными, и в точках $\theta_{G}(x) \neq \theta_{j}, x \in X$, $j=1,2, \ldots, n$, значения $f\left(\theta_{G}(x)\right)$ задаются равенством (2). Рассмотрим случай $\Theta_{0} \cap \Theta \neq \varnothing$ и перенумеруем векторы $\theta_{1}, \ldots, \theta_{n}$ так, что

$$
\Theta_{0} \cap \Theta=\left\{\theta_{1}, \theta_{2}, \ldots, \theta_{k}\right\}, \quad 1 \leqslant k \leqslant n .
$$

Введем обозначения: $F-$ матрица $r \times n, F=\left(f\left(\theta_{1}\right) \vdots \ldots: f\left(\theta_{n}\right)\right), Q$ матрица $p \times n, Q=\left(\theta_{1} \ldots \theta_{n}\right), q^{\mathrm{T}}(x)=\left(q_{1}(x), \ldots, q_{n}(x)\right), l_{j}-n$-мерный столбец с 1 на $j$-м месте, и 0 на всех остальных местах, $j=1,2, \ldots, n, l=\sum_{j=1}^{n} l_{j}$. Пусть

$$
X_{j}=\left\{x \in X: \theta_{G}(x)=\theta_{j}\right\}, \quad j=1,2, \ldots, k .
$$

Тогда в матричном виде

$$
Q\left(q(x)-l_{j}\right)=0, \quad x \in X_{j}, \quad j=1,2, \ldots, k,
$$

и, кроме того, по определению $l^{\mathrm{T}}\left(q(x)-l_{j}\right)=0,1 \leqslant j \leqslant n$, и следовательно,

$$
\widetilde{Q}\left(q(x)-l_{j}\right)=0, \quad x \in X_{j}, \quad j=1,2, \ldots, k,
$$

где $\widetilde{Q}=\left(Q^{\mathrm{T}} \vdots l\right)^{\mathrm{T}}$.

Образуем линейное подпространство $L$, натянутое на векторы $\left\{q(x)-l_{j}, x \in X_{j}\right\}_{j=1}^{k}$. Пусть $d-$ размерность подпространства $L$, a $\widetilde{L}$ - матрица порядка $d \times n$, строки которой являются базисом $L$, т. е. $L=\mathcal{L}(\widetilde{L})$. Очевидно, что $d+v \leqslant n$, где $v=R(\widetilde{Q})$ - ранг матрицы $\widetilde{Q}$. Пусть $Q_{1}$ матриша такая, что $\widetilde{Q} Q_{1}^{\mathrm{T}}=0, L_{1} Q_{1}^{\mathrm{T}}=0$ и $R\left(Q_{1}\right)=n-d-v$, т. е $\mathcal{L}\left(Q_{1}\right)=\mathcal{L}^{\perp}(\widetilde{L}) \cap \mathcal{L}^{\perp}(\widetilde{Q})$, где $\mathcal{L} \perp$ обозначает ортогональное дополнение к соответствующему подпространству. Но из равенства (2) имеем

$$
F\left(q(x)-l_{j}\right)=0, \quad x \in X_{j}, \quad j=1,2, \ldots, k,
$$

а следовательно, $\mathcal{L}(F) \subset \mathcal{L} \perp(\widetilde{L})$, и получаем, что

$$
F=A Q+b e^{\mathrm{T}}+B Q_{1},
$$

где $A$ и $B$ произвольные матрицы порядков $r \times p$ и $r \times R\left(Q_{1}\right)$ соответственно, а $b$ - произвольный $r$-мерный вектор. Переписав (3) в виде

$$
f\left(\theta_{i}\right)=A \theta_{i}+b+B \eta_{i}, \quad i=1,2, \ldots, n,
$$

где $\eta_{1}, \ldots, \eta_{n}$ - столбцы матрицы $Q_{1}$, получаем следующий результат. 
Т е о рем а. Все решения уравнения (2) относительно функции $\mathrm{f}(\cdot)$ имеют вид

$$
f(u)=A u+b+B \sum_{i=1}^{n} \eta_{i} q_{i}(x), \quad x \in X,
$$

где $u \in \Theta_{0} \quad u \quad u=\sum_{i=1}^{n} \theta_{i} q_{i}(x), x \in X$.

С лед с т в ие. $E c л и d+v=n$, то

$$
f(u)=A u+b, \quad u \in \Theta_{0} .
$$

В качестве примера рассмотрим оценивание функции $f$ от среднего $\theta$ в схеме независимых одномерных нормальных наблюдений. Другими словами, в соответствии с обозначениями $p=r=1, X=R^{k}$, $x=\left(x_{1}, \ldots, x_{k}\right), \quad p(x, \theta)=(\sqrt{2 \pi} \sigma)^{-k} \exp \left[-\Sigma\left(x_{i}-\theta\right)^{2} / 2 \sigma^{2}\right], \quad \sigma_{k}^{2}>0$, $d \mu(x)=d x$. В силу достаточности статистики $\bar{x}=\sum_{i=1}^{k} x_{i} / k$ можно принять $k=1$, а кроме того, не умаляя общности, положим $\sigma^{2}=1$. Пусть $\theta_{1}<\theta_{2}<\ldots<\theta_{n}-$ точки сосредоточения распределения $G$, а $p_{1}, \ldots$ $\ldots, p_{n}-$ соответствующие нагрузки. Тогда байесовская оценка $\theta_{G}(x)$ параметра $\theta$ имеет вид, $i=1,2, \ldots n$,

$\theta_{G}(x)=\sum_{i=1}^{n} \theta_{i} q_{i}(x), \quad q_{i}(x)=\exp \left[-\left(x-\theta_{i}\right)^{2} / 2\right] / \sum_{j=1}^{n} \exp \left[-\frac{\left(x-\theta_{j}\right)^{2}}{2}\right]$.

Нетрудно убедиться, что $\theta_{G}$ возрастающая по $x \in R^{1}$ функция, причем область ее значений - открытый интервал $\left(\theta_{1}, \theta_{n}\right)$, и следовательно, $\Theta \cap \Theta_{0}=\left(\theta_{2}, \ldots, \theta_{n-1}\right)$. Обозначим через $x_{2}<\ldots<x_{n-1}$ решение системы

$$
\theta_{G}\left(x_{j}\right)=\theta_{j}, \quad j=2, \ldots, n-1 .
$$

Очевидно, что в силу возрастания $\theta_{G}(x)$ система (5) имеет единственное решение относительно $x_{2}, \ldots, x_{n-1}$, и следовательно, $X_{j}=x_{j}$, $j=2, \ldots, n-1$.

Рассмотрим матрицу

$$
A=\left\{q_{i}\left(x_{j}\right)-\delta_{i j}\right\}_{i=1,2, \ldots, n}^{j=2,3, \ldots, n-1}
$$

и убедимся, что ее ранг равен $n-2$. По теореме Леви-Деспланка $\left[{ }^{1}\right]$, квадратная подматрица $\left\{g_{i}\left(x_{j}\right)-\delta_{i j}\right\} i, j=2, \ldots, n-1$ невырождена, так как $\sum_{i=2}^{n-1} q_{i}\left(x_{j}\right)<1$, и следовательно, ранг матрицы $A$ есть $n-2$. Осталось заметить, что матрица

$$
\left(\begin{array}{ccc}
\theta_{1} & \ldots & \theta_{n} \\
1,1, & \ldots & 1
\end{array}\right)
$$

имеет ранг 2, и значит, выполнено условие следствия к теореме. Таким образом, только линейная функция сохраняет байесовское свойство относительно конечного дискретного априорного распределения при оценивании функции от среднего нормального закона.

\section{Л И Т Е РА Т У Р А}

1. Маркус М., Минк Х. Обзор по теории матриц и матричных неравенств. М., «Наука», 1972.

Ннститут кибернетики Академии наук Эстонской ССР
Поступила в редакцию 4/X 1986 
HINNANGUTE. TEISENDUSE BAYESI OMADUSE SÄILIVUS APRIOORSETE DISKREETSETE MOOTUDE KORRAL

Statistiliste hinnangute teoorias tekib sageli vajadus hinnata mitte tundmatut jaotuse parameetrit $\theta$ ennast, vaid temast sõltuvat funktsiooni $f(\theta)$. Sellistes olukordades on kasulik teada, kas parameetri $\theta$ hinnangu $\hat{\theta}$ mingi statistiline omadus säilib $f(\theta)$ hindamisel statistikuga $f(\hat{\theta})$. Artiklis on iseloomustatud selliseid teisendusi; mis säilitavad hinnangu Bayesi omaduse lõplikus arvus punktides kontsentreeritud aprioorse diskreetse mõõdu suhtes ruutkaofunktsiooni korral. On näidatud, et ühemõõtmelise normaaljaotuse keskväärtuse hindamisel sellised teisendused ammenduvad lineaarfunktsiooni klassiga.

\section{OLMAN}

\section{INVARIANCE OF BAYES PROPERTY SUBJECT TO DISCRETE MEASURE UNDER TRANSFORMATIONS}

In many problems arising in the theory of statistical estimation the value necessary to estimate is not directly the unknown parameter $\theta$ of the considered distribution but some transformation $f(\theta)$ of it. The question considered in the paper may be, in the most general way, stated as follows: for what kind of transformations $f$ some given statistical property of the estimator $\hat{\theta}$ of the parameter $\theta$ is preserved when $f(\theta)$ is estimated by $f(\hat{\theta})$. In this paper a Bayes property of estimator is in question under quadratic risk subject to a priori discrete measure, concentrated in finite numbers of points. The characterization of transformations which are invariant in respect to Bayes property is obtained. It is shown that for normal case these transformations are only linear. 